\title{
Hybrid metal grid-polymer-carbon nanotubes electrodes for high $\underline{\text { luminance organic light emitting diodes }}$
}

F. Laurent M. Sam, G. Dinesha M. R. Dabera, Khue T. Lai, Christopher A. Mills, Lynn J. Rozanski and S. Ravi P. Silva*

Advanced Technology Institute, Department of Electronic Engineering, University of Surrey, Guildford, Surrey, GU2 7XH, UK

*corresponding author: s.silva@surrey.ac.uk

Keywords: OLEDs, metal grids, ITO alternatives, hybrid transparent conducting electrodes.

\section{Abstract}

Organic light emitting diodes (OLEDs) incorporating grid transparent conducting electrodes (TCEs) with wide grid line spacing suffer from an inability to transfer charge carriers across the gaps in the grids to promote light emission in these areas. High luminance OLEDs fabricated using a hybrid transparent conducting electrode (TCE) composed of poly(3,4-ethylenedioxythiophene) poly(styrenesulfonate) (PEDOT:PSS PH1000) or regioregular poly(3-hexylthiophene)-wrapped semiconducting single-walled carbon nanotubes (rrP3HT-SWCNT) in combination with a nanometre thin gold grid are reported here. OLEDs fabricated using the hybrid gold grid/PH1000 TCE have a luminance of $18,000 \mathrm{~cd} / \mathrm{m}^{2}$ at $9 \mathrm{~V}$; the same as the reference indium tin oxide (ITO) OLED. The gold $\mathrm{grid} / \mathrm{rrP3HT}$-SWCNT OLEDs have a lower luminance of $8,260 \mathrm{~cd} / \mathrm{m}^{2}$ at $9 \mathrm{~V}$, which is likely due to a rougher $\mathrm{rP} 3 \mathrm{HT}$-SWCNT surface. These results demonstrate that the hybrid gold grid/PH1000 TCE is a promising replacement for ITO in future plastic electronics applications including OLEDs and organic photovoltaics (OPVs). For applications where surface roughness is not critical, e.g. electrochromic devices or discharge of static electricity, the gold grid/rrP3HT-SWCNT hybrid TCE can be employed. 


\section{Introduction}

Great strides have been made in the development of organic optoelectronic devices such as organic photovoltaics (OPVs) and organic light emitting diodes (OLEDs) [1]. An important part of these devices is the transparent conducting electrode (TCE) which provides a window for light transfer while maintaining electrical contact to the active layers. A good TCE must have a combination of low sheet resistance and high light transmission. The most common TCE at the moment is indium tin oxide (ITO) which has been extensively optimised and characterised [2]. However, due to its scarcity, indium is expensive, and ITO itself is brittle and can have long term issues with indium $[3,4]$ and oxygen $[5,6]$ migration degrading the device.

Alternative TCEs have been developed to replace ITO. Some of the most promising candidates are poly(3,4-ethylenedioxythiophene) poly(styrenesulfonate) (PEDOT:PSS) [7, 8], silver nanowires (AgNW) [9], graphene [10, 11] and carbon nanotubes (CNTs) [12]. Devices have been fabricated using these materials as the $\operatorname{TCE}[7,10,11]$ or as hole injection or extraction layers $[13,14,15,16]$. They have low resistances and can form uniform thin films with high transmission. However, a major problem with these materials is that the resistances remain low only over a short distance because the contact resistance between individual AgNW, CNT, PEDOT:PSS or graphene grains is very high and this limits their conductivity over large areas. For example, CNTs are renowned for almost ballistic intra-tube electrical conduction, but the resistance between CNTs in close physical contact ranges from $50 \mathrm{k} \Omega$ to $3 \mathrm{M} \Omega[17,18]$. Similar problems occur with AgNWs and graphene flakes, whereas PEDOT:PSS has been optimised so that high conductivity versions are available where contact resistance is not as significant, but the sheet resistance is still higher than ITO. One way to overcome this problem is to combine these nanomaterials with a nanometre thin metal grid which is itself a good TCE and has been used to fabricate devices $[19,20]$. The thin metal grid does not suffer from high contact resistance as it is a continuous metal network. Similar grids have also been shown to retain its high conductivity during bending on flexible substrates [21]. 
To ensure a high transmission, the metal grid TCE has traditionally incorporated large grid line spacings $[22,23]$. However, as the line spacing increases beyond the diffusion length of the charge carriers, dark areas appear in OLEDs between the grid lines. Charges injected from the grid into the low conductivity charge transport layers fail to reach the centres of the spaces between grid lines, and therefore recombination with charge carriers injected from the counter electrode occurs only in the vicinity of the grid lines. If the conductive nanomaterials (CNTs, graphene or PEDOT:PSS) are combined with a thin metal grid [23], the total contact resistance will be minimal as relatively few grain boundaries are present. The metal grid allows for conduction over large areas and the conductive nanomaterials spread the current across the grid spaces so that the entire emissive layer (EML) emits light evenly with no dark areas.

This concept has been used previously to produce a hybrid gold grid/graphene TCE which had a good combination of sheet resistance and transmission (20 $\Omega / \square$ and $90 \%)$, but no devices were made [23]. Here, we extend the concept to real device applications to fabricate OLEDs using two types of hybrid TCEs: gold grid/PEDOT:PSS (PH1000, 5\% sorbitol) and gold grid/regioregular poly(3-hexylthiophene) wrapped semiconducting single wall carbon nanotubes (rrP3HT-SWCNT). These OLEDs demonstrate performances comparable to ITO-based OLEDs, especially at low voltages, and clearly indicate their credentials as alternative TCEs in large area electronics.

\section{Experimental Procedure}

Hexagonal gold grids ( $40 \mathrm{~nm}$ thick, $60 \mu \mathrm{m}$ line spacing, $2 \mu \mathrm{m}$ line width) were fabricated on glass substrates $\left(15 \times 15 \times 0.7 \mathrm{~mm}^{3}\right)$ by photolithography and metal sputtering. All the TCEs, including the ITO reference, were deposited on identical glass substrates. To improve PEDOT:PSS wetting, the TCE was plasma cleaned (100 W, 5 minutes) using an oxygen gas flow of $15 \mathrm{sccm}$ (KM1050X Plasma Etcher/Asher/Cleaner, Quorum Technologies). As the wetting is improved, the polymer layer 
thickness was not affected by the surface nanotexture of the grid on the glass substrate. It was found that silver was damaged by the plasma ashing, so gold was chosen instead. It is an inert metal and has a very low resistivity $\left(2.44 \times 10^{-8} \Omega / \square\right)$. The grid dimensions were calculated using a simple simulation to give a sheet resistance of $27 \Omega / \square$ and transmission of $94 \%$ (see Supplementary Materials), comparable to the ITO properties. The polymer layers had to be the same thickness as the ITO OLED, so planarisation layers were not used. This meant that the grid could not be thicker than $40 \mathrm{~nm}$. It was found that thicker grid lines short-circuited the device. The line width was at the limit of the photolithography resolution. As a result, it was found that a line spacing of $60 \mu \mathrm{m}$ gave a TCE with a good combination of sheet resistance and transmission.

The encapsulated OLEDs $\left(2 \times 4 \mathrm{~mm}^{2}\right)$ had the following structure: Glass/ TCE/ PEDOT:PSS (AI4083)/ Poly[(9,9-dioctylfluorenyl-2,7-diyl)-co-(4,4'-(N-(p-butylphenyl))diphenylamine)] (TFB)/ Poly[(9,9-di-noctylfluorenyl-2,7-diyl)-alt-(benzo[2,1,3]thiadiazol-4,8-diyl)] (F8BT)/ 2,9-dimethyl-4,7-diphenyl-1,10phenanthroline (BCP)/ aluminium (Al). All OLED layers were spin coated, except for BCP and Al which were evaporated.

Sorbitol (5 wt\%) was added to PEDOT:PSS PH1000 (Heraeus, Clevios) (this solution will henceforth be called PH1000-S). It was spin coated on to the gold grids and annealed in air ( $\left.150{ }^{\circ} \mathrm{C}, 10 \mathrm{mins}\right)$. A glycerol inter-layer was used to prevent PEDOT:PSS AI4083 from dissolving the PH1000-S layer [24]. This was not necessary for the gold grid/rrP3HT-SWCNT TCE as the deposited rrP3HT-SWCNTs are not water soluble.

The Transmission Line Method (TLM) was used to measure the sheet resistance of PH1000-S and rrP3HT-SWCNT. Their transmission (300 - $1000 \mathrm{~nm}$ wavelength) was measured using a UV-VIS spectrometer (Cary 5000, Agilent Technologies). The substrate contribution is accounted for by placing a blank substrate in the reference beam in the UV-VIS spectrometer. The transmission is therefore measured for the TCE only. Infrared spectroscopy was conducted at $300 \mathrm{~K}$ using a Fourier transform Infrared spectrometer (FTIR, Varian Carey 660). The resolution was $8 \mathrm{~cm}^{-1}$ and the scan 
size was 500. Samples for FTIR were deposited onto polished semi-insulating gallium arsenide (GaAs) substrates. An ammeter/voltage source meter (Keithley 2425) was used to measure the OLED current - voltage characteristics, and a silicon photodiode (Hamamatsu S1227-1010BQ) was used to measure the luminance.

\section{Results and Discussion}

The sheet resistance and transmission of the PH1000-S were measured for different spin speeds, with constant spin time and acceleration. PH1000-S spun at a high spin speed forms a thinner film with higher sheet resistance and transmission (Figure 1). The thickness of the PH1000-S film ranged from $46-60 \mathrm{~nm}$ as the spin speed decreased from $7000 \mathrm{rpm}$ to $3000 \mathrm{rpm}$. The transmission at a wavelength of $550 \mathrm{~nm}$ varies from $93.5-95.5 \%$ depending on the spin speed. The sheet resistance drops from $240 \Omega / \square$ to $180 \Omega / \square$ as the spin speed decreases. A film with the lowest sheet resistance was desired, and therefore a spin speed of $3000 \mathrm{rpm}(1 \mathrm{~min}$ ) was chosen. PH1000-S spun at lower spin speeds did not form a uniform film.

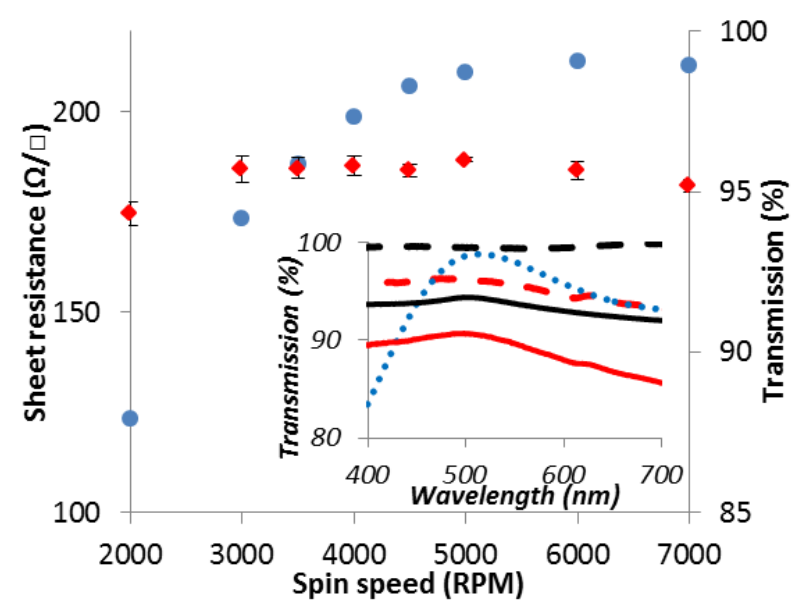

Figure 1. Sheet resistance (blue dots) and transmission (red diamonds) of PH1000-S spin-coated at different spin speeds. Inset- transmission spectra of PH1000-S (3000 rpm) (red dashed line), rrP3HT-SWCNT (1500 rpm) (black dashed line), $40 \mathrm{~nm}$ gold grid/PH1000-S (solid red line), $40 \mathrm{~nm}$ 
gold grid/rrP3HT-SWCNT (solid black line) and ITO (dotted blue line). PH1000-S and $40 \mathrm{~nm}$ gold grid/PH1000-S have a lower transmission than rrP3HT-SWCNT and $40 \mathrm{~nm}$ gold grid/rrP3HT-SWCNT. The gold grid has the same transmission as the gold grid/rrP3HT-SWCNT TCE (overlaps it).

A major consideration for rrP3HT-SWCNT films is surface roughness. If the density of the deposited rrP3HT-SWCNT is too high, then the rough surface will short circuit the device. A low concentration ( $0.025 \mathrm{mg} / \mathrm{ml}$ in dichlorobenzene) spun at a low spin speed (1500 rpm for $1 \mathrm{~min}$ ) was therefore chosen. The sheet resistance of the rrP3HT-SWCNT film was $5.4 \mathrm{M} \Omega / \square$, and the transmission was greater than $99 \%$ for wavelengths of $400-700 \mathrm{~nm}$. ITO has a sheet resistance of $15 \Omega / \square$ and an average transmission of $87 \%$ in the same wavelength range.

$40 \mathrm{~nm}$ gold grid, gold grid/PH1000-S and gold grid/rrP3HT-SWCNT TCEs had sheet resistances of 27, 24 and $25 \Omega / \square$ respectively. The gold grid had a lower sheet resistance than either PH1000-S or rrP3HT-SWCNT on their own. Nevertheless, the sheet resistance of the PH1000-S and rrP3HT-SWCNT in the gaps in the grid is low enough (across the short distance) for the injected charges to spread evenly. This is demonstrated below where OLEDs produced using the gold grids alone displayed dark spots in the gaps between the grid lines, but the light emission was uniform when the PH1000-S or rrP3HT-SWCNT layers were used.

It is known that the addition of sorbitol (or a similar polyalcohol) to PEDOT:PSS of any grade reduces its sheet resistance $[25,26]$. This is due to changes in the morphology of PEDOT:PSS during annealing, resulting in less insulating PSS domains between the PEDOT grains. As a result, the contact resistance between PEDOT grains is reduced. As the annealing temperature $\left(150^{\circ} \mathrm{C}\right)$ is between the melting point $\left(95^{\circ} \mathrm{C}\right)$ and the boiling point $\left(296^{\circ} \mathrm{C}\right)$ of sorbitol, sorbitol evaporates during annealing, which means that the sorbitol is not directly conducting any charge in the polymer. Here, we used FTIR spectroscopy (Figure 2) to show that sorbitol is no longer present in the PEDOT:PSS film after 
annealing, in agreement with Nardes et al. who used mass spectrometry [25] and Jönsson et al. who used X-ray photoelectron spectroscopy (XPS) [27] to prove this.

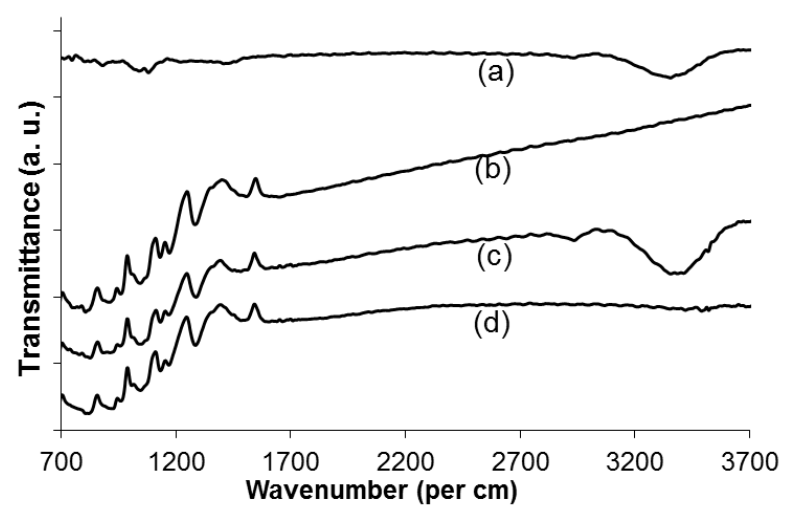

Figure 2. FTIR spectra of (a) pure sorbitol (not annealed), (b) PH1000 (annealed), (c) PH1000-S (before annealing), and (d) PH1000-S (after annealing).

The features observed in Figure 2(a) are similar to those reported for sorbitol [28]. The pre-anneal IR spectrum of PH1000-S (c) and that for sorbitol (a) show peaks at 2,915 (C-H stretching modes) and $3,368 \mathrm{~cm}^{-1}$ (-OH stretching vibrations) due to the presence of sorbitol in the $\mathrm{PH} 1000-\mathrm{S}$. These peaks disappear after annealing (d), indicating that the annealed PH1000-S is completely devoid of sorbitol molecules. The features observed from 700 to $1,700 \mathrm{~cm}^{-1}$ in (b) and (d) are similar to those reported for PH1000 [29, 30].

The rrP3HT-SWCNT network formed a uniform layer on the substrate (Figure 3(a)) with a low density of rrP3HT-SWCNT $\left(2 \pm 0.6 \mathrm{rrP3HT}\right.$-SWCNT per $\left.\mu \mathrm{m}^{2}\right)$ and low surface roughness (root-mean squared (RMS) roughness of $2.0 \pm 0.4 \mathrm{~nm}$ ). The rrP3HT-SWCNT layer is about 1-2 CNTs thick. PH1000-S is slightly smoother with a RMS roughness of $1.6 \pm 0.6 \mathrm{~nm}$ (Figure 3(b)). The RMS roughness on both sides of the grid line in Figure $3(\mathrm{c})$ is $0.71 \pm 0.04 \mathrm{~nm}$. A good network of rrP3HT-SWCNT is formed so that no point on the surface is further than $0.5 \mu \mathrm{m}$ from a rrP3HT-SWCNT allowing current to spread uniformly across the surface. PH1000-S on the other hand has no spaces and completely covers the 
substrate surface. In the microscope image of the gold grid only OLED (Figure 5(b)), it can be seen that the holes can move about $6 \mu \mathrm{m}$ away from the grid lines (through PEDOT:PSS AI4083). The 0.5 $\mu \mathrm{m}$ gaps between individual rrP3HT-SWCNTs can easily be covered by the holes, so the light emission can be uniform.
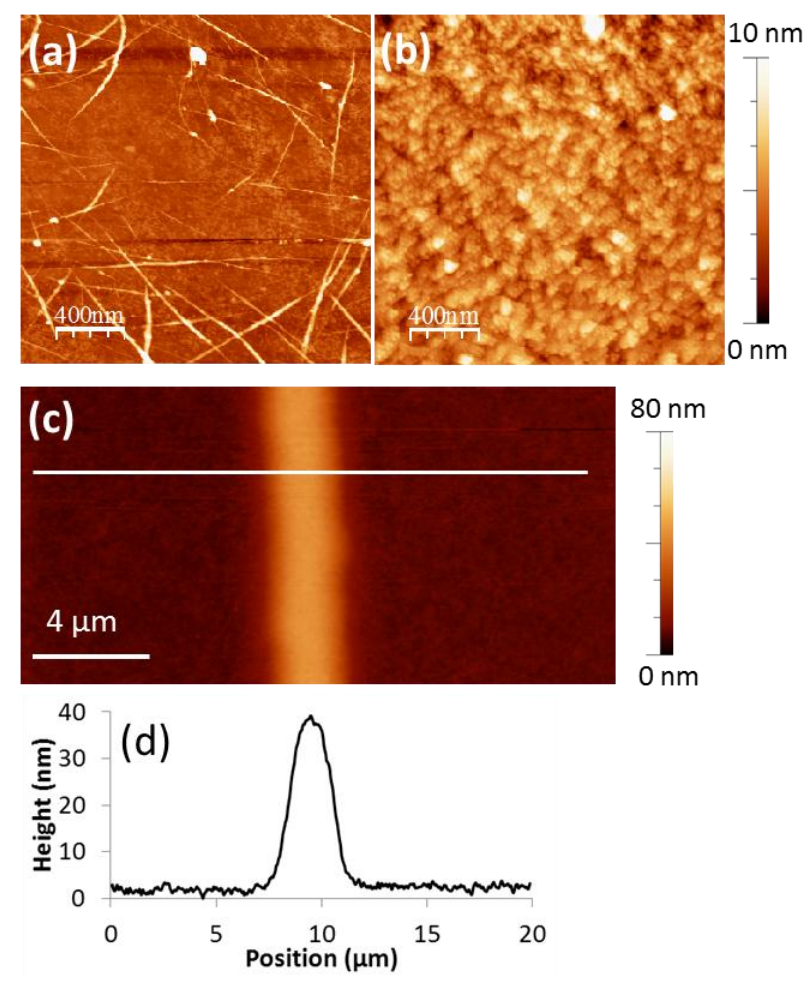

Figure 3. AFM height images of (a) rrP3HT-SWCNT and (b) PH1000-S deposited on glass. (c) Larger scan size showing the grid line covered by PH1000-S. Grid line covered by rrP3HT-SWCNT shows a similar image. (d) Surface profile of the hybrid grid TCE along the position shown by the white line in (c).

One OLED was fabricated on a $45 \mathrm{~nm}$ thick gold grid/PH1000-S (90 \% transmission and $24 \Omega / \square$ sheet resistance), and another on a $41 \mathrm{~nm}$ thick gold grid/rrP3HT-SWCNT (94\% and $25 \Omega / \square)$. Ideally the gold grid should be the same thickness for both OLEDs, but there is always some variation in the sputtered metal thickness. There is only a small difference in sheet resistance and optical transmission between 41 and $45 \mathrm{~nm}$ thick gold grid ( $25 \Omega / \square$ versus $24 \Omega / \square$, and $94.1 \%$ versus 
94.0\%). The largest effect on the performance of the OLED (compared to using a simple grid TCE) is expected to be from PH1000-S or rrP3HT-SWCNT.

The performance of the OLEDs using the hybrid TCEs is significantly better in terms of luminance than a TCE made of just the grid or just PH1000-S on its own, and is comparable to an ITO OLED (Figure 4). The maximum luminance of the gold grid OLED is $5,300 \mathrm{~cd} / \mathrm{m}^{2}$ at $20.0 \mathrm{~V}$, even though its sheet resistance and transmission are similar to ITO. The gold grid/rrP3HT-SWCNT TCE OLED has an improved maximum luminance of $14,900 \mathrm{~cd} / \mathrm{m}^{2}$ at $12.5 \mathrm{~V}$, whilst the gold grid/PH1000-S TCE OLED has a maximum luminance of $23,100 \mathrm{~cd} / \mathrm{m}^{2}$ at $12.5 \mathrm{~V}$. For comparison, the ITO OLED has a maximum luminance of $26,200 \mathrm{~cd} / \mathrm{m}^{2}$ at $12.0 \mathrm{~V}$. A PH1000-S OLED (without a grid TCE) had a maximum luminance of $12,000 \mathrm{~cd} / \mathrm{m}^{2}$ at $25.0 \mathrm{~V}$ due to its high sheet resistance (Supplementary Materials, Figure S1).
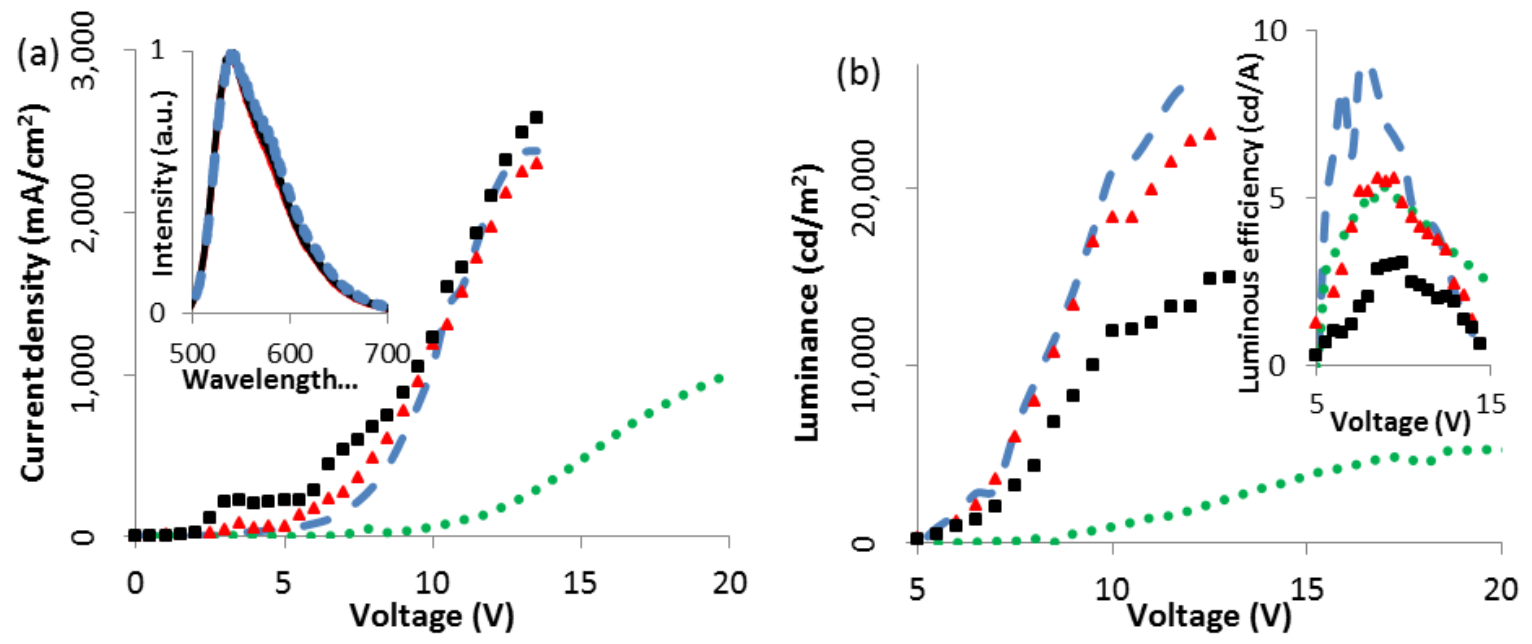

Figure 4. OLED performance with different hybrid TCEs. (a) Current density versus voltage, (inset (a)) OLED emission spectra, (b) luminance versus voltage, (inset (b)) luminous efficiency versus voltage. Black squares $-41 \mathrm{~nm}$ gold grid/rrP3HT-SWCNT, red triangles $-45 \mathrm{~nm}$ gold grid/PH1000-S, blue dashes - ITO reference device, green dots $-40 \mathrm{~nm}$ gold grid only. 
The relative luminances of the OLEDs can be linked to their uniformity of light emission. The gold grid has large spacings between the grid lines to maintain a high transmission (Figure 5(a)), but this results in dark areas in the gaps (Figure $5(b)$ ) and therefore low luminance. A conductive material such as PH1000-S or rrP3HT-SWCNT, helps to conduct charge across these gaps, so that recombination occurs over the whole area of the EML (Figures $5(c)$ and (d)), the light emission is homogeneous and the luminance is higher as a result.

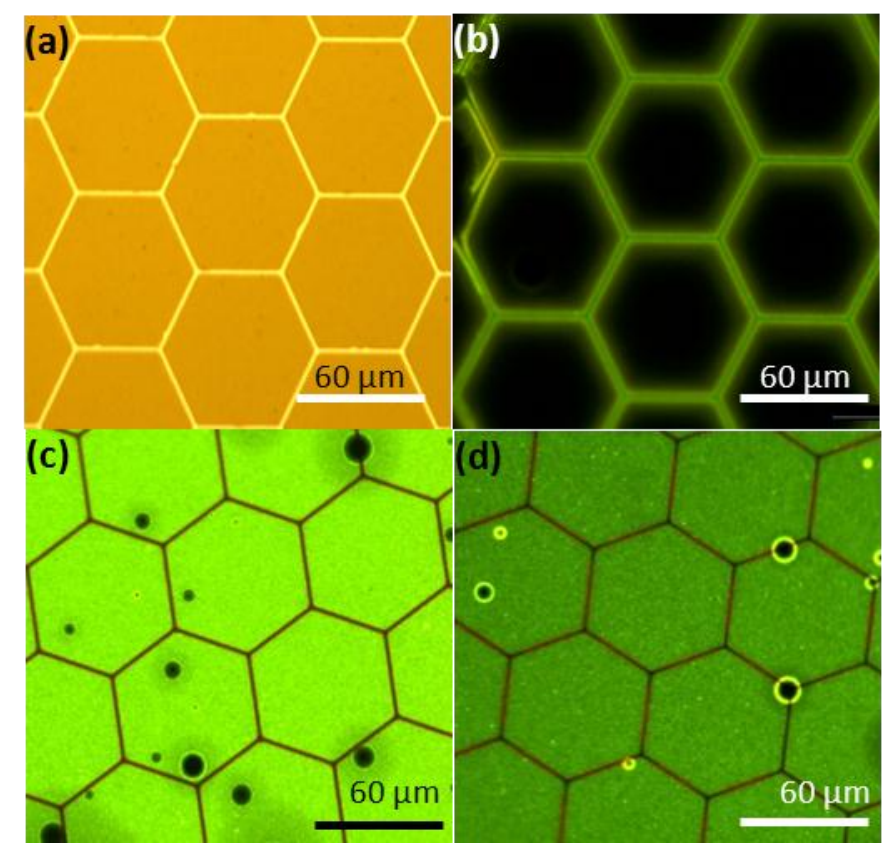

Figure 5. Light microscope images of (a) a gold grid only TCE OLED (off), (b) a gold grid only TCE OLED (on), (c) a gold grid/PH1000-S TCE OLED (on), (d) a gold grid/rrP3HT-SWCNT TCE OLED (on). The light microscope lamp was turned off to take images (b) - (d). OLEDs were powered using a $9 \mathrm{~V}$ battery.

The dark spots seen in Figure 5(c) and (d) are intrinsic to all the OLEDs produced, including the reference ITO OLEDs. The lifetime of the hybrid grid OLEDs have not been measured, but they last as long as the reference ITO OLEDs. It is not clear what causes dark spots to occur and as yet there is no conclusive explanation for them in the literature. It could be due to processing conditions, 
particulate exposure or material failures during operation. Insufficient dark spots are seen in Figure 5 to conclusively say that they occur mostly near or on the grid lines, and examination of other areas suggests that the distribution is random. Similar dark spots have been seen previously for OLEDs where cathode delamination is identified as the cause [31]. Elsewhere, exposure to particulates [32] and atmospheric oxygen and water [33] have also been cited. Anode effects have not tended to be cited as the cause [31]. Improved cathode deposition and encapsulation techniques should mitigate for these defects and improve device lifetime.

Although the hybrid TCEs have a slightly lower transmission than ITO (Figure 1 (inset)), they have very similar luminances. The gaps in the grid are covered by PH1000-S or rrP3HT-SWCNT only, so the transmission in the gaps is $96 \%$ and $99 \%$ respectively, which is as good as for ITO. This is the case for $93.5 \%$ of the surface are of the hybrid grid. The rest of the area is covered by a metal grid. This blocks the light incident on the grid and reflects it back to the aluminium cathode. The light can then reflect back from the cathode and out through the gaps in the grid. Only a very small percentage is absorbed by the metal or the polymer layers. The corrugated surface of the grid can also aid in the extraction of light [34] which might otherwise be trapped and waveguided in the polymer layers if the TCE was flat. The high reflectivity of the electrodes, and the improved extraction of light compensates for the slightly lower transmission, and the luminance of the hybrid gold grid/PH1000S TCE OLEDs is therefore the same as the ITO OLED.

The hybrid TCE OLEDs attain 100 and $1,000 \mathrm{~cd} / \mathrm{m}^{2}$ (the typical luminances of a computer screen and a fluorescent lamp respectively) at similar voltages to the ITO OLED (Table 1). Therefore, it can be concluded that the performance of the gold grid/PH1000-S and gold grid/rrP3HT-SWCNT OLEDs are comparable to the ITO OLED at low voltages making them a good alternative to ITO. At higher luminance above $1,000 \mathrm{~cd} / \mathrm{m}^{2}$, the gold grid/rrP3HT-SWCNT OLED is not as bright as the ITO or gold grid/PH1000-S OLEDs. The luminance of the gold grid/PH1000-S OLED is nearly identical to the reference ITO OLED up to $9 \mathrm{~V}$ (luminance $=18,000 \mathrm{~cd} / \mathrm{m}^{2}$ ) after which the luminance of the gold 
Accepted for publication in Nanotechnology (Institute of Physics)

grid/PH1000-S OLED rises at a slower rate. It could be that at high voltage and long operating times, heat builds up in the OLED and degrades the PH1000-S earlier than the ITO or gold grid which are more resistant to high temperatures.

\begin{tabular}{c|c|c|c}
\hline & \multicolumn{3}{|c}{ TCE } \\
\hline & ITO & $\begin{array}{c}\text { Gold grid/ } \\
\text { PH1000-S }\end{array}$ & $\begin{array}{c}\text { Gold grid/ } \\
\text { rrP3HT-SWCNT }\end{array}$ \\
\hline $\begin{array}{c}\text { Voltage to } \\
\text { achieve } \\
100 \mathrm{~cd} / \mathbf{m}^{2} \text { (V) }\end{array}$ & 5.1 & 4.7 & 4.7 \\
\hline $\begin{array}{c}\text { Voltage to } \\
\text { achieve 1,000 } \\
\text { cd/m } \mathbf{m}^{\mathbf{2}} \text { (V) }\end{array}$ & 5.5 & 5.9 & 6.1 \\
\hline \hline
\end{tabular}

Table 1. Voltage required for the ITO, hybrid gold grid/PH1000-S and gold grid/rrP3HT-SWCNT TCE OLEDs to operate at 100 and $1,000 \mathrm{~cd} / \mathrm{m}^{2}$.

The transmissions of the gold grid and the hybrid TCEs are consistently above $90 \%$ over the visible wavelength range. rrP3HT-SWCNT do not preferentially absorb specific wavelengths (Figure 1(inset)). PH1000-S has a slightly lower transmission at longer wavelengths. However, between 500 and 700 $\mathrm{nm}$, the transmission varies between 90 and $94 \%$. This variation is so small and the transmission is so high that it also gives the impression of being colour neutral so that the emission spectra of the gold grid/PH1000-S and gold grid/rrP3HT-SWCNT OLEDs are the same as the ITO OLED (inset Figure 4(a)). ITO has a peak transmission at $515 \mathrm{~nm}$, which is close to the F8BT peak emission wavelength $(540 \mathrm{~nm})$, but it has a greater variation in transmission with wavelength than the hybrid TCEs. As a result, for red, blue and white OLEDs, the hybrid grids may produce a better performance than ITO.

Although the rrP3HT-SWCNT film has a low RMS roughness, it is still rougher than the PH1000-S film. As a result, there is a higher leakage current in the gold grid/rrP3HT-SWCNT OLED than in the gold grid/PH1000-S or ITO OLEDs. This is evident by the higher current density in the gold grid/rrP3HTSWCNT OLED, especially at voltages below the turn-on voltage (Figure 4(a)). The radiative recombination of holes and electrons is less efficient when the leakage current is high, and the 
luminance and luminous efficiency are correspondingly lower. The maximum luminous efficiency (inset Figure 4(b)) of the gold grid/rrP3HT-SWCNT, gold grid/PH1000-S, gold grid only, PH1000-S only and ITO OLEDs are 3.1, 5.6, 5.4, 6.0 and $9.1 \mathrm{~cd} / \mathrm{A}$, respectively. Although the gold grid/PH1000-S OLED has the same luminance as the ITO OLED, it has a lower efficiency. The reason for the lower efficiency is unclear at present. It is possible that the gold grid/PH1000-S OLED luminous efficiency is largely determined by the $\mathrm{PH} 1000-\mathrm{S}$ (which also has a lower luminous efficiency) as it covers most of the area. There could be a charge imbalance in the OLED due to the double layer of PEDOT:PSS, which changes the charge recombination area in the EML. If this is the case, then the thickness of the polymer layers can be optimised in future to rebalance the charges for the hybrid TCE OLEDs and increase their efficiency.

\section{Conclusion}

We have demonstrated OLEDs with good performance characteristics using hybrid TCEs composed of a thin gold grid and PH1000-S or rrP3HT-wrapped SWCNT. Although the transmission and sheet resistance of the hybrid grid is mostly unchanged from the pristine gold grid, the two components of the hybrid grid combine to make a brighter OLED than either the gold grid, PH1000-S or rrP3HTSWCNT on their own. At voltages below $9 \mathrm{~V}$, the luminance of the gold grid/PH1000-S OLED is almost the same as that of an ITO OLED, and the gold grid/PH1000-S and gold grid/rrP3HT-SWCNT OLEDs reach $1,000 \mathrm{~cd} / \mathrm{m}^{2}$ at a similar voltage as an ITO OLED. The gold grid/rrP3HT-SWCNT OLED does not show as high a luminance as the ITO OLED, due to a high surface roughness leading to high leakage currents. This can be solved by improving the rrP3HT-SWCNT deposition process to produce smoother films. If this can be achieved and cheaper metals such as copper are used, the performance of the grid/rrP3HT-SWCNT TCE OLED may become comparable to an ITO OLED, with the added advantages that the TCE will be stronger, more flexible and cheaper to produce than ITO. 


\section{Acknowledgements}

K. T. Lai would like to thank R. Gwilliam, and M. D. Lourenco for the supply and preparation of the semi-insulating GaAs substrates for the FTIR measurements.

\section{References}

[1] Forrest S R 2003 Org. Electron. 4 45-8

[2] Kim H, Gilmore C M, Pique A, Horwitz I S, Mattoussi H, Murata H, Kafafi Z H and Chrisey D B 1999 J. Appl. Phys. 86 6451-61

[3] Aziz H and Popovic Z D 2004 Chem. Mater. 16 4522-32

[4] Sheats J R and Roitman D B 1998 Synth. Met. 95 79-85

[5] Lo M F, Ng T W, Mo H W and Lee C S 2013 Adv. Funct. Mater. 23 1718-23

[6] Lo M F, Ng T W, Lai S L, Fung M K, Lee S T and Lee C S 2011 Appl. Phys. Lett. 99033302

[7] Havare A K, Can M, Demic S, Kus M and Icli S 2012 Synth. Met. 161 2734-8

[8] Alshammari A S, Shkunov M and Silva S R P 2014 Phys. Status Solidi RRL 8 150-3

[9] Tokuno T, Nogi M, Jiu J and Suganuma K 2012 Nanoscale Res. Lett. 7281

[10] Wang X, Zhi L and Mullen K 2008 Nano Lett. 8 323-7

[11] Becerril H A, Mao J, Liu Z, Stoltenberg R M, Bao Z and Chen Y 2008 ACS Nano 2 463-70

[12] Wu Z, Chen Z, Du X, Logan J M, Sippel J, Nikolou M, Kamaras K, Reynolds J R, Tanner D B, Hebard A F and Rinzler A G 2004 Science 305 1273-6

[13] Inigo A R, Underwood J M and Silva S R P 2011 Carbon 49 4211-7

[14] Ou E C W, Hu L, Ruey G C, Soo O K, Pan J, Zheng Z, Park Y, Hecht D, Irvin G, Drzaic P and Gruner G 2009 ACS Nano 3 2258-64

[15] Shi S and Silva S R P 2012 Carbon 50 4163-70 
[16] Dabera G D M R, Jayawardena K D G I, Prabath M R R, Yahya I, Tan Y Y, Nismy N A, Shiozawa H, Sauer M, Ruiz-Soria G, Ayala P, Stolojan V, Adikaari A A D T, Jarowski P D, Pichler T, and Silva S R P 2013 ACS Nano 7 556-565

[17] Buldum A and Lu J P 2001 Phys. Rev. B 63161403

[18] An L. and Friedrich C. R., Nucl. Instr. Meth. Phys. Res. B 272 169-172

[19] Galagan Y, Rubingh J E J, Andriessen R, Fan C C, Blom P W, Veenstra S C and Kroon J M 2011 Sol. Energ. Mat. Sol. Cells 95 1339-43

[20] Sam F L M, Mills C A, Rozanski L J and Silva S R P 2014 Laser Photon. Rev. 8 172-9

[21] Hong S, Yeo J, Kim G, Kim D, Lee H, Kwon J, Lee H, Lee P and Ko S H 2013 ACS Nano 7 5024-31

[22] Kuang P, Park J M, Leung W, Mahadevapuram R C, Nalwa K S, Kim T G, Chaudhary S, Ho K M and Constant K 2011 Adv. Mater. 23 2469-73

[23] Zhu Y, Sun Z, Yan Z, Jin Z and Tour J M 2011 ACS Nano 5 6472-9

[24] Tseng S R, Meng H F, Yeh C H, Lai H C, Horng S F, Liao H H, Hsu C S and Lin L C 2008 Synth. Met. $158130-4$

[25] Nardes A M, Kemerink M, de Kok M M, Vinken E, Maturova K and Janssen R A J 2008 Org. Electron. 9 727-34

[26] Lai S L, Chan M Y, Fung M K, Lee C S and Lee S T 2003 Mat. Sci. Eng. B-Solid 104 26-30

[27] Jonsson S K M, Birgerson J, Crispin X, Greczynski G, Osikowicz W, Denier van der Gon A W, Salaneck W R and Fahlman M 2003 Synth. Met. 139 1-10

[28] Kaviya S, Santhanalakshmi J and Viswanathan B 2011 J. Nanotechnol. 2011, 2011152970

[29] Nagarajan S, Kumar J, Bruno F F, Samuelson L A and Nagarajan R 2008 Macromolecules 413049 52

[30] Jian J, Guo X, Lin L, Cai Q, Cheng J and Li J 2013 Sens. Actuator B-Chem 178 279-288

[31] Liew Y F, Aziz H, Hu N X, Hardy S O C, Xu G and Popovic Z 2000 Appl. Phys. Lett. 772650

[32] Lim S F, Wang W and Chua S J 2001 Mater. Sci. Eng. B 85 154-9

[33] Chwang A B, Rothman M A, Mao S Y, Hewitt R H, Weaver M S, Silvernail J A, Rajan K, Hack M, Brown J J, Chu Xi, Moro L, Krajewski T and Rutherford N 2003 Appl. Phys. Lett. 83413

[34] Hobson P A, Wedge S, Wasey J A E, Sage I and Barnes W L 2002 Adv. Mater. 14 1393-6 
Accepted for publication in Nanotechnology (Institute of Physics) 